By

\title{
Antonio DiCarlo
}

IMA Preprint Series \# 2045

(May 2005)

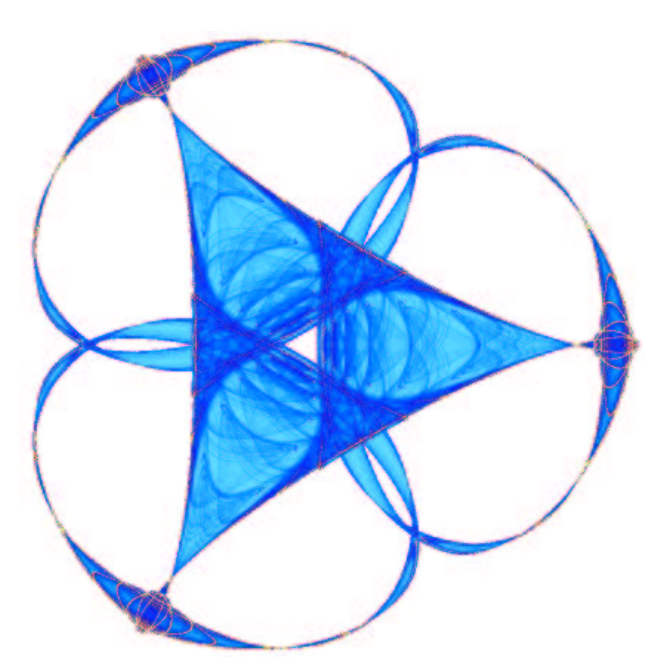

INSTITUTE FOR MATHEMATICS AND ITS APPLICATIONS UNIVERSITY OF MINNESOTA

514 Vincent Hall

206 Church Street S.E.

Minneapolis, Minnesota 55455-0436

Phone: 612/624-6066 Fax: 612/626-7370

URL: http://www.ima.umn.edu 


\title{
Surface and Bulk Growth Unified
}

\author{
Antonio DiCarlo*
}

May 20, 2005

\section{Contents}

1 Introduction 2

2 A continuum theory of bulk growth 3

2.1 Kinematics . . . . . . . . . . . . . . . 4

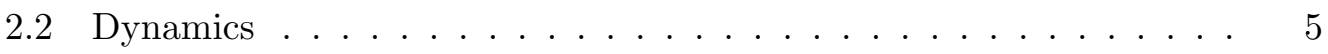

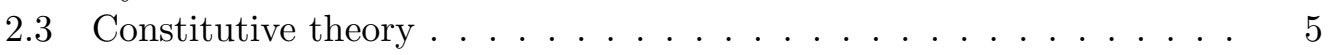

$\begin{array}{lll}3 & \text { Piecewise compatible bulk growth } & 7\end{array}$

$\begin{array}{llr}4 & \text { Coherent interfaces } & 9\end{array}$

$\begin{array}{llr}5 & \text { Concluding remarks } & 10\end{array}$

\section{Notice}

This paper is due to appear in the book Mechanics of Material Forces (Proceedings of the homonymous EuroMech Colloquium held in Kaiserslautern, Germany, May 21-24, 2003) edited by P. Steinmann and G.A. Maugin, to be published in the Springer Series "Advances in Mechanics and Mathematics." The manuscript was first submitted to the editors on July 30, 2003, and finally accepted in its present form on July 21, 2004.

Solid surfaces can have their physical area changed in two ways, either by creating or destroying surface without changing surface structure and properties per unit area, or by an elastic strain ... along the surface keeping the number of surface lattice sites constant.... .

—J.W. Cahn, 1980

*SMFM@DiS: Mathematical Structures of Materials Physics, Dept. of Studies on Structures, Università degli Studi "Roma Tre", Via Vito Volterra 62, I-00146 Roma (Italy) 〈adicarlo@mac.com〉. 


\begin{abstract}
I have been puzzled for a long time by the unnatural divide between the theory of bulk growth — strikingly underdeveloped — and that for surface growth — much better developed, along apparently independent lines.

Recent advances in growth mechanics (DiCarlo and Quiligotti, 2002) make it now possible to subsume growth phenomena of both kinds under one and the same format, where surface growth is obtained as an infinitely intense bulk growth confined in a layer of vanishingly small thickness.

This has allowed me to recover the results collected in Gurtin (2000) from the standpoint of DiCarlo and Quiligotti (2002). In particular, I am able to construe Gurtin's technique of referential control volumes that evolve in time as a special application of the principle of virtual power.
\end{abstract}

\title{
1 Introduction
}

Any continuum theory of growth - be it modelled as spread in bulk or concentrated on surfaces - hinges on two key issues: in kinematics, extra degrees of freedom have to be introduced, in order to distinguish growth from deformation; in dynamics, new balance laws have to be provided, apt to govern the evolution of such degrees of freedom.

Both issues are much subtler for bulk than for surface growth, however, demanding a sharper veering from the customary route in continuum mechanics. In my mind, here lies the basic difficulty which hindered surface and bulk growth theories from progressing on a par, within a common frame.

The theory of bulk growth which I (still) prefer is the one set forth by DiCarlo and Quiligotti (2002). ${ }^{1}$ It is the only one I am aware of where the evolution law for bulk growth is obtained as a constitutively augmented balance. In order to make this paper reasonably self-contained, Sect. 2 offers a résumé of that theory, adapted to the present discussion.

As a paradigm for surface growth theories, I take the tract by Gurtin (2000): it is a convenient reference book, where the role of an independent configurational balance is duly stressed - from a point of view, I may add, which in several respects differs from my own.

\footnotetext{
${ }^{1}$ This is not at all obvious, since I can change my mind. But in this respect I did not. My coauthor did (Quiligotti, 2002), on the (not minor) issue of invariance requirements, convinced by an argument intimated by Green and Naghdi (1971) and spelled out in Casey and Naghdi (1980, 1981), Casey (1987), and elsewhere. For reasons I expound elsewhere (DiCarlo, in preparation), I find that old argument faulty and misleading.
} 
The quote from Cahn (1980) I put in the prologue is a paraphrase of Gibb's discussion of multiphase equilibria. I took it from the introductory chapter of Gurtin (2000), where it is completed by the following comment from the author: "The creation of surface involves configurational forces, while stretching the surface involves standard forces." Despite the fact that Gibbs, Cahn and Gurtin had only surface phenomena in mind (namely, the evolution of phase interfaces), it is perfectly legitimate - and much to the point - to rephrase the whole statement in terms of bulk phenomena, as follows (italicized words are my own):

Solid bodies can have their physical volume changed in two ways, either by creating or destroying bulk without changing bulk structure and properties per unit volume, or by an elastic strain within the body keeping the number of bulk lattice sites constant. The creation of bulk involves configurational couples, while stretching the body involves standard forces.

By couple I mean a tensor quantity - not necessarily skew - having the physical dimensions of length $\times$ force. Why configurational couples are germane to bulk growth is explained in Sect.2.2; explaining how they match with configurational forces, proper to surface growth, is one of the main aims of this paper.

\section{A continuum theory of bulk growth}

The growing bodies considered here are standard Cauchy continua: the only kinematic descriptor ascribed to their points is place in ordinary physical space. In order to distinguish growth from deformation, two evolving configurations are associated with each body element: its current configuration, describing how it is actually placed in space, and its relaxed configuration, describing how it "would like" to be placed. The field of relaxed configurations need not be (and usually is not) compatible, not even locally.

This is a good old kinematic idea, primarily introduced to distinguish between elastic and viscoplastic strains by Kröner (1960) and Lee (1969), and much later imported into growth modelling by Rodriguez et al. (1994) (see also Taber (1995)). The original contribution by DiCarlo and Quiligotti (2002) is in dynamics. As summarized in the following, we obtain the evolution law for bulk growth as a constitutively augmented new balance, the balance of configurational (or remodelling) couples, independent of the standard force balance. 


\section{$2.1 \quad$ Kinematics}

We regard a body as a smooth manifold $\mathcal{B}$ (with boundary $\partial \mathcal{B}$ ), and call placement any smooth embedding

$$
p: \mathcal{B} \rightarrow \mathcal{E}
$$

of the body into the Euclidean place manifold $\mathcal{E}$, whose translation space will be denoted by $V \mathcal{E}$. Tangent vectors on the body manifold itself are called line elements. The set of all line elements attached to a single body-point $b \in \mathcal{B}$ is called the body element at $b$, and denoted $\mathrm{T}_{b} \mathcal{B}$ (the tangent space to $\mathcal{B}$ at $b$ ). The union of all body elements is denoted $\mathrm{TB}$ (the tangent bundle of $\mathcal{B}$ ).

The body gradient $\nabla p$ of a placement $p$ is a tensor field on $\mathcal{B}$, whose value at any given point $b$, denoted by $\left.\nabla p\right|_{b}$, maps linearly the body element $\mathrm{T}_{b} \mathcal{B}$ onto $\mathrm{VE}$. We call stance any tensor field of this kind, be it a gradient or not. Therefore, a stance is any smooth mapping

$$
\mathrm{P}: \mathrm{TB} \rightarrow \mathrm{VE}
$$

such that the restriction $\mathrm{P} \mid \mathrm{T}_{b} \mathcal{B}$ is a linear embedding, for all $b \in \mathcal{B}$. If a stance happens to be the gradient of a placement, we say that it is induced by that placement: all placement induces a stance, but a general stance is not induced by any placement, not even locally. We describe growth by the time evolution of the relaxed stance $\mathbb{P}$, while motion is described as the time evolution of the actual placement $p$.

The complete motion of a growing body is a family of pairs $(p, \mathbb{P})$ smoothly parametrized by the time line $\mathcal{T}$ (identified with the real line), and the velocity realized along that motion at the time $\tau \in \mathcal{T}$ is the pair of fields (a superposed dot denoting time differentiation):

$$
\left(\dot{p}(\tau), \dot{\mathbb{P}}(\tau) \mathbb{P}(\tau)^{-1}\right): \mathcal{B} \rightarrow \mathrm{V} \mathcal{E} \times(\mathrm{V} \mathcal{E} \otimes \mathrm{VE}) .
$$

The linear space of test velocities $\mathfrak{T}$, comprising all smooth fields

$$
(\mathrm{v}, \mathbb{V}): \mathcal{B} \rightarrow \mathrm{VE} \times(\mathrm{V} \mathcal{E} \otimes \mathrm{VE}),
$$

will play a central role in the next subsection. The visible velocity of body-points (with physical dimensions length/time) is given by the vector field $\mathrm{v}$, while the tensor field $\mathbb{V}$ gives the growth velocity of the corresponding body elements (with physical dimensions $1 /$ time). 


\subsection{Dynamics}

To us, a force is primarily a continuous linear real-valued functional on the space of test velocities, whose value we call the working expended by that force. ${ }^{2}$ We assume that the total working expended on any test velocity $(\mathrm{v}, \mathbb{V}) \in \mathfrak{T}$ admits the following integral representation:

$$
\int_{\mathcal{B}}-(\mathrm{s} \cdot \mathrm{v}+\mathbb{C} \cdot \mathbb{V}+\mathbb{S} \cdot \mathrm{Dv})+\int_{\mathcal{B}}(\mathrm{b} \cdot \mathrm{v}+\mathbb{B} \cdot \mathbb{V})+\int_{\partial \mathcal{B}} \mathrm{t}_{\partial \mathcal{B}} \cdot \mathrm{v}
$$

where the integrals are taken with respect to the relaxed volume and surface area of body elements, and D denotes the relaxed gradient:

$$
\mathrm{Dv}:=(\nabla \mathrm{v}) \mathbb{P}^{-1}
$$

Because of the compound structure of test velocities (4), the force functional splits additively into a brute force, dual to $\mathrm{v}$, and a remodelling force, dual to $\mathbb{V}$. Another important splitting is between the inner working, given by the first bulk integral in (5), and the outer working, given by the remaining sum. The brute self-force per unit volume s, the outer brute bulk-force per unit volume b, and the brute boundary-force per unit area $t_{\partial \mathcal{B}}$ take values in $V \mathcal{E}$; the remodelling self-couple per unit volume $\mathbb{C}$, the brute Piola stress $\mathbb{S}$ (also a specific couple!), and the outer remodelling couple per unit volume $\mathbb{B}$ take values in $V \mathcal{E} \otimes V \mathcal{E}$.

All balance laws are systematically provided by the principle of null working: the total working expended on any test velocity should be zero, i.e., the total force should be the null functional. Skipping the balance of brute forces, which is standard, I present here only the balance of remodelling couples:

$$
-\mathbb{C}+\mathbb{B}=0 \quad \text { on } \mathcal{B} \text {. }
$$

\subsection{Constitutive theory}

Our treatment of constitutive issues rests on two pillars (altogether independent of balance): the principle of material indifference to change in observer, and the

\footnotetext{
${ }^{2}$ The physical dimensions of the working are God given: energy/time. Since the prototypal space of test velocities is VE, with physical dimensions length/time, forces get typically identified with elements of VE itself, with physical dimensions energy/length (= "force" by definition!). As velocities have not dimensions length/time, in general (think of growth velocity $\mathbb{V}$ ), a general force has not dimensions "force". Another force related issue is that the space $\mathfrak{T}$ of test velocities should be endowed with the structure of a topological vector space, so as to make meaningful the physically essential requirement that force functionals be continuous. I leave gaps like this to be filled in by the mathematically conscious reader.
} 
dissipation principle. Both of them deliver strict selection rules on the constitutive prescription for the inner force. Such a priori restrictions do not apply to the outer force, which is regarded as an adjustable control on the process. This inner/outer dichotomy does not pertain to the physics of interactions, but to the limitations of the model: what appears as an outer interaction within a given theory may always be accounted for - in principle - as an inner interaction within a broader and more cumbersome theory. In an all-embracing model there would be no outer interactions at all. In our model of growth mechanics, the outer remodelling couple $\mathbb{B}$ has a determinative role whenever growth — be it surface or bulk - is powerfully controlled by non-mechanical phenomena, such as biochemical reactions in living tissues.

I will only flash the outcome of the first principle, as applied by DiCarlo and Quiligotti (2002), while summarizing with some more detail the machinery of the second one. Material indifference rules out non-trivial values of the brute self-force $\mathrm{s}$ and non-symmetric values of the Cauchy stress $\mathrm{T}:=|\operatorname{det} \mathrm{F}|^{-1} \mathbb{S F}^{\top}$, where the warp

$$
\mathrm{F}:=\mathrm{D} p=(\nabla p) \mathbb{P}^{-1} \text {. }
$$

measures how the actual stance, i.e., the body gradient of the actual placement, differs from the relaxed stance. If we further assume that the response of the body element at $b$ filters off from $(p, \mathbb{P})$ all information other than $\left.p\right|_{b},\left.\nabla p\right|_{b}$, and $\left.\mathbb{P}\right|_{b}$, we obtain from the same principle that

$$
\mathbb{S}(b, \tau)=\mathrm{R}(b, \tau) \check{\mathbb{S}}_{b}\left(\left.\mathrm{U}\right|_{b},\left.\mathbb{P}\right|_{b}, \tau\right), \quad \mathbb{C}(b, \tau)=\check{\mathbb{C}}_{b}\left(\left.\mathrm{U}\right|_{b},\left.\mathbb{P}\right|_{b}, \tau\right),
$$

the rotation $\mathrm{R}$ and the stretch $\mathrm{U}$ being, respectively, the orthogonal and the right positive-symmetric factor of the warp (8): $\mathrm{F}=\mathrm{RU}$.

To have a notion of dissipation, an additional energetic descriptor is needed. We postulate the existence of an additive real-valued free energy $\Psi(\mathcal{P})=\int_{\mathcal{P}} \psi$, measuring the inner energy available to body-parts. Since we take integrals over body-parts with respect to the relaxed volume, $\psi$ represents the free energy per unit relaxed volume. We call power expended along a process at time $\tau$ the opposite of the working expended by the inner force due to the process on the velocity realized at time $\tau$. Hence, the power expended measures the working done by an outer force balanced with the constitutively determined inner force. The dissipation principle we enforce requires that the power dissipated - defined as the difference between the power expended along a process and the time derivative of the free energy - should be non-negative, for all body-parts, at all times. ${ }^{3}$ This localizes into:

$$
\dot{\psi}+\psi \mathrm{I} \cdot \mathbb{V} \leq \mathbb{S} \cdot \mathrm{Dv}+\mathbb{C} \cdot \mathbb{V},
$$

\footnotetext{
${ }^{3}$ Due account is to be taken of the fact that the relaxed-volume form, let's say $\mu$, evolves in time, as dictated by the growth velocity $\mathbb{V}=\dot{\mathbb{P P}}^{-1}: \dot{\Psi}(\mathcal{P})=\left(\int_{\mathcal{P}} \psi \mu\right)^{\circ}=\int_{\mathcal{P}}(\psi \mu)^{\circ}=\int_{\mathcal{P}}(\dot{\psi} \mu+\psi \dot{\mu})=$ $\int_{\mathcal{P}}(\dot{\psi}+\psi \mathrm{I} \cdot \mathbb{V}) \mu(\mathrm{I}$ denotes the identity on $\mathrm{VE} ; \mathrm{I} \cdot \mathbb{V}$ is the trace of $\mathbb{V})$.
} 
it being intended that $\mathrm{v}$ and $\mathbb{V}$ are given by (3), $\mathbb{S}$ and $\mathbb{C}$ by (9), and $\psi$ has to be related to the process by an extra constitutive mapping. Our main constitutive assumption selects a rather special, but very interesting constitutive class, beautifully accounted for by Epstein (1999). We posit that, at each body-point, the present value of the free energy per unit relaxed volume depends only on the present value of the warp at that point:

$$
\psi(b, \tau)=\check{\psi}_{b}(\mathrm{~F}(b, \tau)) .
$$

The requirement that (10) be satisfied along all processes is fulfilled if and only if for each $b$ (which will be dropped from now on) the responses $\breve{\mathbb{S}}$ and $\check{\mathbb{C}}$ satisfy ( $\partial$ denotes differentiation):

$$
\check{\mathbb{S}}=\partial \check{\psi}+\stackrel{+}{\mathbb{S}}, \quad \check{\mathbb{C}}=\mathbb{E}+\stackrel{+}{\mathbb{C}},
$$

with

$$
\mathbb{E}:=\check{\psi} \mathrm{I}-\mathrm{F}^{\top} \check{\mathbb{S}}
$$

the Eshelby coupling between brute mechanics and remodelling, and the extraenergetic responses $\stackrel{+}{\mathbb{S}}, \stackrel{+}{\mathbb{C}}$ restricted by the reduced dissipation inequality

$$
\stackrel{+}{\mathbb{S}} \cdot \dot{\mathrm{F}}+\stackrel{+}{\mathbb{C}} \cdot \mathbb{V} \geq 0
$$

to be abided by in the same sense as (10). As is seen, the Eshelbian coupling is mandatory (within the constitutive class we are considering) and independent of any special assumption on $\check{\mathbb{S}}$. Additional couplings - through the outer remodelling couple $\mathbb{B}$, in particular - are not ruled out.

It should be stressed that $\mathbb{B}$, while trivial in most - if not all-applications to "dead" engineering materials, plays a major role even in the simplest biomechanical applications, where it describes the mechanical feedback from the biochemical control system: think of so-called stress-dependent growth laws (Taber, 1995). Therefore, the idea - emphasized by Epstein and Maugin (2000) - that the Eshelby coupling is the "driving force of irreversible growth" is untenable for any smartly controlled material. The Eshelby coupling by itself does not drive any smart growth; rather, it drives a dull - though nontrivial - visco-plastic flow, as shown by DiCarlo, Nardinocchi and Teresi (forthcoming).

\section{$3 \quad$ Piecewise compatible bulk growth}

Let $\mathfrak{C}_{\mathrm{P}}$ be a collection of open disjoint patches, whose closures cover the body manifold $\mathcal{B}$, and $\mathfrak{C}_{\mathrm{F}}$ the corresponding collection of interfaces. In order to cover also the 
body boundary $\partial \mathcal{B}$ with (closures of) interfaces in $\mathfrak{C}_{\mathrm{F}}$, it is convenient to augment $\mathfrak{C}_{\mathrm{P}}$ with an idle patch $\mathcal{P}_{\text {ext }}$, the exterior of $\mathcal{B}$.

Now, enforce a local compatibility constraint on the relaxed stance $\mathbb{P}$, requiring that on each patch $\mathcal{P} \in \mathfrak{C}_{\mathrm{P}}$ there is a local placement

$$
\boldsymbol{p}_{\mathcal{P}}: \mathcal{P} \rightarrow \mathcal{E}
$$

smooth up to $\partial \mathcal{P}$, such that

$$
\mathbb{P} \mid \mathcal{P}=\nabla \boldsymbol{p}_{\mathcal{P}} .
$$

Parallelly, restrict the testing of the remodelling force to piecewise compatible growth velocities, i.e., on tensor fields $\mathbb{V}$ that, on each patch $\mathcal{P} \in \mathfrak{C}_{\mathrm{P}}$, are the relaxed gradient of a vector field $\mathbf{w}_{\mathcal{P}}$, smooth up to $\partial \mathcal{P}$ :

$$
\mathbb{V} \mid \mathcal{P}=D \mathbf{w}_{\mathcal{P}}=\left(\nabla \mathbf{w}_{\mathcal{P}}\right) \mathbb{P}^{-1} .
$$

Then, denoting by $\mathbb{A}:=\mathbb{B}-\mathbb{C}$ the total remodelling couple per unit relaxed volume $\left(\mathbb{A}=0\right.$ in $\left.\mathcal{P}_{\text {ext }}\right)$, the working expended on $\mathbb{V}$ is given by

$$
\begin{aligned}
\int_{\mathcal{B}} \mathbb{A} \cdot \mathbb{V} & =\sum_{\mathcal{P} \in \mathfrak{C}_{\mathrm{P}}^{j}} \int_{\mathcal{P}} \mathbb{A} \cdot \mathbb{V}=\sum_{\mathcal{P} \in \mathfrak{C}_{\mathrm{P}}} \int_{\mathcal{P}} \mathbb{A} \cdot\left(\mathrm{Dw}_{\mathcal{P}}\right) \\
& =\sum_{\mathcal{P} \in \mathfrak{C}_{\mathcal{P}}}\left(\int_{\partial \mathcal{P}}\left(\mathbb{A} \mathbf{n}_{\partial \mathcal{P}}\right) \cdot \mathbf{w}_{\mathcal{P}}-\int_{\mathcal{P}}(\operatorname{Div} \mathbb{A}) \cdot \mathbf{w}_{\mathcal{P}}\right),
\end{aligned}
$$

with $\mathbf{n}_{\partial \mathcal{P}}$ the outward unit normal to the relaxed shape of $\partial \mathcal{P}$. Hence, the principle of null working yields the following balances: for each $\mathcal{P} \in \mathfrak{C}_{\mathrm{P}}$,

$$
\begin{array}{ll}
\operatorname{Div}(\mathbb{E}+\stackrel{+}{\mathbb{C}}-\mathbb{B})=0 & \text { on } \mathcal{P}, \\
(\mathbb{E}+\stackrel{+}{\mathbb{C}}-\mathbb{B}) \mathbf{n}_{\partial \mathcal{P}}=0 & \text { on } \partial \mathcal{P} .
\end{array}
$$

This set compares with, but is weaker than, $(7)$ plus $\left(9_{2}\right)$ and $\left(12_{2}\right)$.

Eq. (19a) matches with the configurational force balance (5-10) on page 37 of Gurtin (2000), provided that his configurational stress $\boldsymbol{C}$ is identified with $\mathbb{E}$-as implied by the correspondence between (13) and the Eshelby relation (6-9) on page 43 -, and his configurational body forces, internal $\boldsymbol{g}$ and external $\boldsymbol{e}$, are related to my inner and outer remodelling couples by

$$
\boldsymbol{g} \leftarrow \operatorname{Div} \stackrel{+}{\mathbb{C}}, \quad \boldsymbol{e} \leftarrow-\operatorname{Div} \mathbb{B}
$$

Here and in the following, assignment statements such as

$$
q_{\text {surf }} \leftarrow T q_{\text {bulk }}
$$


should be read as meaning that the constitutive assignment for quantity $q_{\text {surf }}$ in surface-growth theory is obtained as the $T$-image of the constitutive assignment for quantity $q_{\text {bulk }}$ in bulk-growth theory.

Analogously, (19b) agrees (modulo an obvious change in notation) with the interfacial force balance $\left(7.7_{2}\right)$ given by Cermelli and Gurtin (1994, Part B: theory of incoherent interfaces without interfacial structure), provided that their interfacial configurational forces, internal $\mathbf{e}_{\partial \mathcal{P}}$ and external $\boldsymbol{f}_{\partial \mathcal{P}}$, are assigned according to

$$
\mathbf{e}_{\partial \mathcal{P}} \leftarrow \stackrel{+}{\mathbb{C}} \mathbf{n}_{\partial \mathcal{P}}, \quad \boldsymbol{f}_{\partial \mathcal{P}} \leftarrow-\mathbb{B} \mathbf{n}_{\partial \mathcal{P}}
$$

Imitating Gurtin (2000), I will now concentrate on coherent interfaces.

\section{Coherent interfaces}

Interface coherency is obtained by enforcing the further constraint that the local relaxed placement in any two adjoining patches $\mathcal{P}_{+}, \mathcal{P}_{-}$be continuous across the interface $\mathcal{S}:=\partial \mathcal{P}_{+} \cap \partial \mathcal{P}_{-}:$the jump across $\mathcal{S}, \llbracket \boldsymbol{p} \rrbracket_{\mathcal{S}}:=\boldsymbol{p}_{\mathcal{S}}^{+}-\boldsymbol{p}_{\mathcal{S}}^{-}$(the limit from $\mathcal{P}_{+}$ minus that from $\mathcal{P}_{-}$), should vanish. If test velocities are analogously restricted by the condition $\llbracket \mathbf{w} \rrbracket_{\mathcal{S}}=0$ on all $\mathcal{S} \in \mathfrak{C}_{\mathrm{F}}$, then the integrals over patch boundaries in (18) yield

$$
\sum_{\mathcal{P} \in \mathfrak{C}_{\mathrm{P}}} \int_{\partial \mathcal{P}}\left(\mathbb{A} \mathbf{n}_{\partial \mathcal{P}}\right) \cdot \mathbf{w}_{\mathcal{P}}=-\sum_{\mathcal{S} \in \mathfrak{C}_{\mathrm{F}}} \int_{\mathcal{S}}\left(\llbracket \mathbb{A} \rrbracket_{\mathcal{S}} \mathbf{m}_{\mathcal{S}}\right) \cdot \mathbf{w}_{\mathcal{S}},
$$

where $\mathbf{w}_{\mathcal{S}}:=\mathbf{w}_{\mathcal{S}}^{+}=\mathbf{w}_{\mathcal{S}}^{-}, \mathbf{m}_{\mathcal{S}}:=\mathbf{n}_{\partial \mathcal{P}_{-}}=-\mathbf{n}_{\partial \mathcal{P}_{+}}$(pay attention to signs and draw a sketch!). Hence, on each $\mathcal{S} \in \mathfrak{C}_{\mathrm{F}}$,

$$
\llbracket \mathbb{E}+\stackrel{+}{\mathbb{C}}-\mathbb{B} \rrbracket_{\mathcal{S}} \mathbf{m}_{\mathcal{S}}=0
$$

in accord with the configurational force balance at the interface (11-8b) on page 68 of (Gurtin, 2000), provided his interface configurational forces, internal $\boldsymbol{g}^{\mathcal{S}}$ and external $\boldsymbol{e}^{\mathcal{S}}$, be given by

$$
\boldsymbol{g}^{\mathcal{S}} \leftarrow \llbracket \stackrel{+}{\mathbb{C}} \rrbracket_{\mathcal{S}} \mathbf{m}_{\mathcal{S}}, \quad \boldsymbol{e}^{\mathcal{S}} \leftarrow-\llbracket \mathbb{B} \rrbracket_{\mathcal{S}} \mathbf{m}_{\mathcal{S}} .
$$

To obtain a genuine surface-growth theory, the growth process should be further specialized, confining the realized growth velocity $\mathbb{V}=\dot{\mathbb{P}}^{-1}$ (cf. (3)) in thin layers around interfaces. For $\varepsilon>0$ small enough, let

$$
\mathcal{L}_{\varepsilon}:=\left\{\boldsymbol{p}_{\mathcal{S}}(b)+\zeta \mathbf{m}_{\mathcal{S}}(b) \mid b \in \mathcal{S},-\varepsilon<\zeta<\varepsilon\right\}
$$


be the $\varepsilon$-thickening of the relaxed shape of an interface $\mathcal{S}$, and $\mathcal{L}_{\varepsilon}^{+}, \mathcal{L}_{\varepsilon}^{-}$its upper $(\zeta>0)$ and lower $(\zeta<0)$ halves, respectively. Assume then (my definition of choice for tensor multiplication implying that $(\mathbf{m} \otimes \mathbf{w}) \mathbf{r}=(\mathbf{m} \cdot \mathbf{r}) \mathbf{w})$ :

$$
\mathbb{V}= \pm \varepsilon^{-1} \mathbf{m}_{\mathcal{S}} \otimes \mathbf{w}_{\mathcal{S}} \quad \text { on } \mathcal{L}_{\varepsilon}^{\mp}, \quad \mathbb{V}=0 \quad \text { elsewhere. }
$$

Notice that the minus sign prevails in $\mathcal{L}_{\varepsilon}^{+}$, and vice versa: the tensor field (27) is, to within $O(1)$ terms for $\varepsilon \downarrow 0$ on most of $\mathcal{L}_{\varepsilon}$, the relaxed gradient of the vector field given by $(b, \zeta) \mapsto(\varepsilon-|\zeta|) \mathbf{w}_{\mathcal{S}}(b)$ inside $\mathcal{L}_{\varepsilon}$ and vanishing outside it. The remodelling force, when evaluated on test fields having the structure (27), in the limit for $\varepsilon \downarrow 0$ yields a pure surface working:

$$
\lim _{\varepsilon \downarrow 0} \int_{\mathcal{B}} \mathbb{A} \cdot \mathbb{V}=-\sum_{\mathcal{S} \in \mathfrak{C}_{\mathrm{F}}} \int_{\mathcal{S}}\left(\llbracket \mathbb{A} \rrbracket_{\mathcal{S}} \mathbf{m}_{\mathcal{S}}\right) \cdot \mathbf{w}_{\mathcal{S}},
$$

in agreement with (23) and (18), where the bulk integrals appearing in the last sum asymptotically vanish. Eqs. (27) and (28) explain how the remodelling couples dominating bulk growth induce configurational forces - in the sense of Gurtin - in the limit theory of surface growth (cf. (25)).

I now integrate the reduced dissipation inequality (14) over $\mathcal{L}_{\varepsilon}$ and take the limit for $\varepsilon \downarrow 0$, assuming that the brute dissipation per unit volume $\stackrel{+}{\mathbb{S}} \cdot \dot{\mathrm{F}}$ is $O(1)$. I thus obtain the surface dissipation inequality

$$
\left(\llbracket \stackrel{+}{\mathbb{C}} \rrbracket_{\mathcal{S}} \mathbf{m}_{\mathcal{S}}\right) \cdot \mathbf{w}_{\mathcal{S}}=\boldsymbol{g}^{\mathcal{S}} \cdot \mathbf{w}_{\mathcal{S}} \leq 0
$$

(recall $\left(25_{1}\right)$ ). Now, if invariance under reparametrization of the interface $\mathcal{S}$ is required, implying that the working depends only on the (scalar) normal velocity of the interface $V_{\mathcal{S}}:=\mathbf{w}_{\mathcal{S}} \cdot \mathbf{m}_{\mathcal{S}}$, then the internal configurational force on the interface is necessarily normal:

$$
\boldsymbol{g}^{\mathcal{S}}=g^{\mathcal{S}} \mathbf{m}_{\mathcal{S}},
$$

with $g^{\mathcal{S}}$ scalar-valued (cf. (11-11) on page 69 of Gurtin (2000)), and (29) coincides with the interfacial dissipation inequality (11-21) on page 71 of Gurtin (2000): $g^{\mathcal{S}} V_{\mathcal{S}} \leq 0$.

\section{Concluding remarks}

Lack of space prevents me from treating theories with interfacial structure, intersecting interfaces (junctions), or growing cracks (fracture). To allow for interfacial structure, the integral representation of the working (5) should be extended to include measures concentrated on interfaces, both for brute and remodelling forces; 
in (18), this would bring out an extra sum over $\mathfrak{C}_{\mathrm{F}}$ of area integrals over interfaces. Junctions and cracks require concentrations on subsets of higher codimension: the intersection of two or more interfaces, or the crack tip.

As a closing remark, let me quote the introduction to Gurtin (2000): "Indetermination arises in the configurational system whenever there is no change in material structure." This is exactly why the theory of configurational forces, applied to changes in material structure confined to meagre subsets of the body manifold, is fraught with indeterminacy. The bulk balance (19a) (coincident with (5-10) on page 37 of Gurtin (2000)), while much weaker than (7), is altogether superfluous in surface growth, being identically satisfied by a reactive internal body force $\boldsymbol{g}$ (introduced in (20)), which is the reaction to the constraint of no structural change in the bulk. This does not help in advocating the independence and usefulness of the configurational force system. However, bulk growth gives us our revenge.

\section{Acknowledgments}

I am grateful to the colloquium organisers, to the discussants at the colloquium and to the anonymous referee for prompting me, in various ways, to organize my ideas and to write them down. My work was supported by GNFM-INdAM (the Italian Group for Mathematical Physics), and by MIUR (the Italian Ministry of Education, University and Research) through the Project "Mathematical Models for Materials Science."

\section{References}

Cahn, John W. (1980). "Surface stress and the chemical equilibrium of small crystals. 1. The case of the isotropic surface," Act. Metall. 28, 1333-1338.

Casey, James. (1987). "Invariance considerations in large strain elasto-plasticity," ASME J. Appl. Mech. 54, 247.

Casey, James, and Paul M. Naghdi. (1980). "A remark on the use of the decomposition $\mathrm{F}=\mathrm{F}_{e} \mathrm{~F}_{p}$ in plasticity," ASME J. Appl. Mech. 47, 672-675.

Casey, James, and Paul M. Naghdi. (1981). "A correct definition of elastic and plastic deformation and its computational significance," ASME J. Appl. Mech. 48, 683-984. 
Cermelli, Paolo, and Morton E. Gurtin. (1994). "The dynamics of solid-solid phase transitions. 2. Incoherent interfaces," Arch. Rational Mech. Anal. 127, 41-99.

DiCarlo, Antonio (in preparation). Material Remodelling.

DiCarlo, Antonio, Paola Nardinocchi, and Luciano Teresi (forthcoming). "Creep as passive growth."

DiCarlo, Antonio, and Sara Quiligotti. (2002). "Growth and balance," Mech. Res. Comm. 29, 449-456.

Epstein, Marcelo. (1999). "On material evolution laws," in Geometry, Continua $\mathcal{E}$ Microstructure (G.A. Maugin ed.). Paris: Hermann, 1-9.

Epstein, Marcelo, and Gérard A. Maugin. (2000). "Thermomechanics of volumetric growth in uniform bodies," Int. J. Plast. 16, 951-978.

Green, Albert E. and Paul M. Naghdi. (1971). "Some remarks on elastic-plastic deformation at finite strain," Int. J. Engng. Sci. 9, 1219-1229.

Gurtin, Morton E. (2000). Configurational Forces as Basic Concepts of Continuum Physics. Berlin: Springer-Verlag.

Kröner, Ekkehart. (1960). "Allgemeine Kontinuumstheorie der Versetzungen und Eigenspannungen," Arch. Rational Mech. Anal. 4, 273-334.

Lee, Erastus H. (1969). "Elastic-plastic deformations at finite strains," J. Appl. Mechanics 36, 1-6.

Quiligotti, Sara. (2002). "On bulk growth mechanics of solid-fluid mixtures: kinematics and invariance requirements," Theor. Appl. Mech. 28/29, 277-288.

Rodriguez, Edward K., Anne Hoger, and Andrew D. McCulloch. (1994). "Stressdependent finite growth in soft elastic tissues," J. Biomechanics 27, 455-467.

Taber, Larry A. (1995). "Biomechanics of growth, remodeling, and morphogenesis," Appl. Mech. Rev. 48, 487-545. 